\title{
Amniotic fluid embolism with second trimester pregnancy termination: a case report
}

\section{[Embolie amniotique lors d'une interruption de grossesse au deuxième trimestre :} exposé d'un cas]

\author{
Barry K. Ray MD, * Manuel C. Vallejo MD, ${ }^{*}$ Mitchell D. Creinin MD, $†$ Kelly T. Shannon MD, \\ Gordon L. Mandell MD, ${ }^{*}$ Bupesh Kaul MD, ${ }^{*}$ Sivam Ramanathan MD*
}

\begin{abstract}
Purpose: Describe the diagnosis, clinical features, pathophysiology, treatment and anesthetic management of amniotic fluid embolism (AFE) in a patient undergoing second trimester pregnancy termination.

Clinical features: A 30-yr-old gravida 2, para I, woman was admitted for a dilatation and evacuation procedure for underlying intra-uterine fetal demise in her second trimester of pregnancy. Hypotension, shock, respiratory arrest, pulseless electrical activity, hemorrhage, disseminated intravascular coagulopathy, requiring cardiopulmonary resuscitation and blood transfusion complicated her intraoperative care. AFE was considered the most likely cause of this intraoperative event.
\end{abstract}

Conclusions: It is now recognized that the pathophysiological features of AFE are similar to a type-I hypersensitivity reaction ranging from mild systemic reaction to anaphylaxis and shock. AFE has a high maternal and fetal morbidity and mortality rate, requiring prompt recognition and treatment. In patients with cardiovascular instability, the treatment of AFE is similar to anaphylaxis requiring aggressive fluid hydration, cardiopulmonary resuscitation, administration of blood products and the use of vasopressors.

Objectif : Décrire le diagnostic, les éléments cliniques, la physiopathologie, le traitement et les mesures anesthésiques entourant une embolie amniotique (EA) chez une patiente qui a subi une interruption de grossesse au deuxième trimestre.

Éléments cliniques : Une femme de 30 ans, G2 Pl, a été admise pour subir une dilatation et une évacuation pour mort fœetale pendant le deuxième trimestre de la grossesse. Hypotension, choc, arrêt respiratoire, dissociation électromécanique, hémorragie, coagulopathie intravasculaire disséminée nécessitant une réanimation cardio-pulmonaire et une transfusion sanguine ont compliqué les soins peropératoires. L'EA a été considérée comme la cause la plus probable de cet événement peropératoire.
Conclusions : On sait maintenant que les caractéristiques physiopathologiques d'une EA sont similaires à une réaction d'hypersensibilité de type I allant d'une réaction bénigne généralisée à l'anaphylaxie et au choc. L'EA présente un taux élevé de morbidité et de mortalité maternelles et fotales qui nécessitent une identification et un traitement rapides. En cas d'instabilité cardiovasculaire, le traitement de l'EA est semblable à celui de l'anaphylaxie et requiert une hydratation liquide importante, une réanimation cardio-pulmonaire, l'administration de dérivés sanguins et l'usage de vasopresseurs.

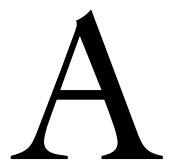

MNIOTIC fluid embolism (AFE) is a rare life-threatening complication unique to pregnancy. AFE has a mortality rate of 61 to $86 \%$ and accounts for approximately $10 \%$ of all maternal deaths in the United States. ${ }^{1,2}$ AFE has a variable presentation, ranging from mild degrees of organ dysfunction to cardiovascular collapse, coagulopathy and death. ${ }^{1,2}$ AFE usually presents at term during labour. ${ }^{3}$ In this report, we present a patient in her second trimester of pregnancy who developed sudden hypoxic respiratory failure followed by cardiopulmonary arrest and coagulopathy following dilatation and evacuation (D \& E) for intra-uterine fetal demise.

\section{Case report}

A 30-yr-old gravida-2, para-1, $99 \mathrm{~kg}, 164 \mathrm{~cm}$, Caucasian woman was admitted for D \& E for an intra-uterine fetal demise at 22 weeks gestation by last menstrual period. A routine ultrasound examination at 18 weeks gestation confirmed the gestational age and demonstrated no fetal abnormalities. A follow-up

From the Departments of Anesthesiology, ${ }^{*}$ Obstetrics, Gynecology and Reproductive Sciences, $†$ Magee Womens Hospital, University of Pittsburgh School of Medicine, Pittsburgh, Pennsylvania, USA. Address correspondence to: Dr. Manuel C. Vallejo, Magee Womens Hospital, 300 Halket Street, Pittsburgh, PA 15213, USA.

Phone: 412-641-4260; Fax: 412-641-4766; E-mail: vallejomc@anes.upmc.edu Accepted for publication May 29, 2003.

Revision accepted November 11, 2003. 
examination was performed four weeks later because of an abnormal maternal multiple marker screen. A fetal demise of approximately 17 weeks gestational size was found, with a normal volume of amniotic fluid. Cervical osmotic dilators were placed the following day, which was one day prior to the $\mathrm{D} \& \mathrm{E}$ procedure.

Her past medical history was significant for a diagnosis of asthma with infrequent albuterol use and drug allergies to penicillin, erythromycin, and cephalexin. She had one full term spontaneous vaginal delivery without complications. Past surgical history was significant for cervical cryosurgery 12 years prior with subsequent PAP smears within normal limits, and two arthroscopic knee surgeries in the remote past. Past anesthetic history was without complications and there was no family history of adverse events related to anesthesia. She had a Mallampati class II airway and physical examination was unremarkable for cardiopulmonary abnormalities.

In a patient with intra-uterine fetal demise, we routinely obtain a preoperative baseline blood coagulation panel when there is a four-week difference between expected gestational age and size. The blood coagulation panel consisting of: fibrinogen; prothrombin time (PT); partial thromboplastin time (PTT); international normalized ratio (INR); hemoglobin and platelet count were normal (Table I). Oxygen $\left(\mathrm{O}_{2}\right)$ was administered via a nasal cannula at 2 $\mathrm{L} \cdot \mathrm{min}^{-1}$ and iv sedation consisting of midazolam 2 $\mathrm{mg}$, fentanyl $100 \mu \mathrm{g}$ and a continuous propofol infusion was given. An intracervical block consisting of $1 \%$ mepivacaine $-20 \mathrm{~mL}$ and vasopressin - five units was administered prior to the procedure. Initial electrocardiogram revealed a normal sinus rhythm.

The procedure was initiated by attempted aspiration of the amniotic fluid; however, no fluid was present. Shortly thereafter, the patient was noted to have paroxysmal coughing, restlessness and peripheral cyanosis. Continuous pulse-oximeter saturation at this point was in the high 90's (Table II). The evacuation procedure was rapidly and easily completed under ultrasound guidance. After evacuation of the uterine contents, a suction aspiration was performed. During the aspiration, the continuous pulse-oximeter saturation dropped from $100 \%$ to the low 80 's despite administering $100 \%$ $\mathrm{O}_{2}$ via facemask (Table II). The patient was noted to have lost consciousness. Subsequent hypoxic respiratory failure ensued and positive pressure bag-mask ventilation was delivered. The electrocardiogram revealed a narrow complex arrhythmia without a pulse [i.e., pulseless electrical activity (PEA), Figure]. Chest compressions were started and endotracheal intubation was achieved on the first attempt. The Advanced Cardiac Life Support (ACLS) protocol for PEA was instituted. Epinephrine $2 \mathrm{mg}$ were given via the endotracheal tube and epinephrine $\mathrm{l} \mathrm{mg}$ and atropine $\mathrm{l} \mathrm{mg}$ were given intravenously, along with rapid crystalloid infusion. At this time period, two additional anesthesiologists and a critical care intensivist assisted in cardiopulmonary resuscitation $(\mathrm{CPR})$. During resuscitation, a right external jugular vein, a right femoral vein triple lumen, and a left femoral arterial line where placed for central access and monitoring. A continuous dopamine infusion followed by a continuous norephinephrine infusion were given via the central access to maintain hemodynamic stability. Intraoperative blood gas analysis revealed; $\mathrm{pH}$ $=7.22, \mathrm{pO}_{2}=252, \mathrm{pCO}_{2}=40.3, \mathrm{HCO}_{3}=16.0$, base excess $=-10$, and an anion gap $=20$, which was consistent with metabolic acidosis. The patient had approximately five minutes of CPR prior to recovery. During $\mathrm{CPR}$, the electrocardiograph revealed an idioventricular arrhythmia, which rapidly deteriorated to ventricular tachycardia, followed by asystole, which spontaneously converted to sinus tachycardia (Figure).

Shortly after the resuscitative efforts were initiated, profuse vaginal bleeding was noted despite a firm uterus. Oxytocin 40 units were added to the lactate Ringer's solution and given over a period of five minutes. Additionally, both methergine $0.2 \mathrm{mg}$ and carboprost tromethamine (Hemabate ${ }^{\circledR}$, Pharmacia Corporation, Kalamazoo, MI, USA) $0.25 \mathrm{mg}$ were given by $i m$ injection. Clinically, the bleeding was consistent with disseminated intravascular coagulopathy (DIC; Table I). Initial arrest laboratory data were sent and not available at the time of intraoperative blood product administration. Three units of packed red blood cells, two units of fresh frozen plasma, and two units of cryoprecipitate were given in the operating room. The patient was taken to the intensive care unit (ICU) intubated and hemodynamically stable on a norepinephrine infusion at $0.02 \mu \mathrm{g} \cdot \mathrm{kg}^{-1} \cdot \mathrm{min}^{-1}$.

The patient remained coagulopathic in the ICU (Table I). Based on laboratory data, an additional two units of packed red blood cells, eight units of fresh frozen plasma and 14 units of cryoprecipitate were given to treat the DIC. Chest radiograph revealed mild bilateral congestion, a minimal left pleural effusion, and two rib fractures, likely resulting from CPR.

On postoperative day one, her condition markedly improved. She was extubated $14 \mathrm{hr}$ after resuscitation and weaned off pressor support. Her coagulopathy resolved (Table I) and the patient was transferred to the step down unit later that same day. The remainder of her hospital stay was unremarkable except for a lowgrade fever (maximal temperature of $38^{\circ} \mathrm{C}$ ) that was 


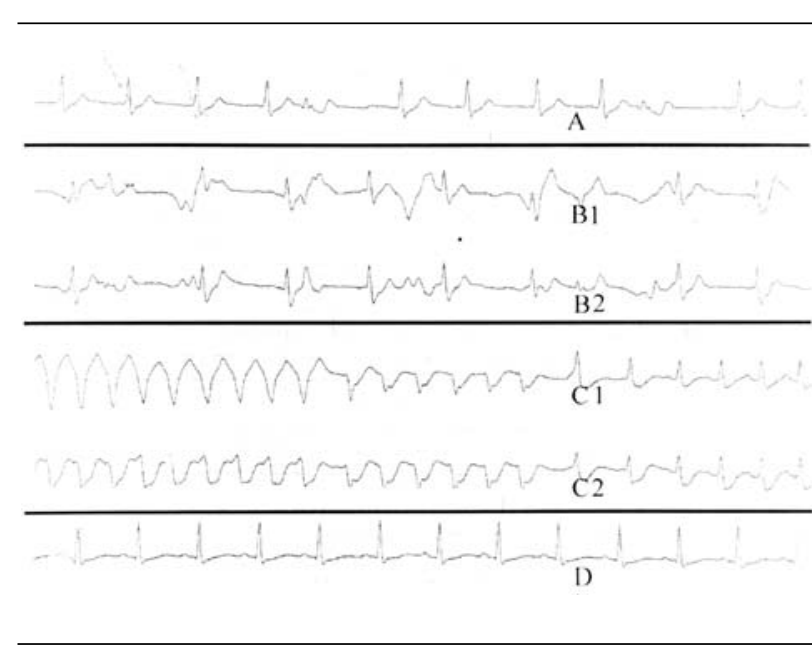

FIGURE Intraoperative electrocardiograms

$\mathrm{A}=$ narrow complex arrhythmia in lead $\mathrm{V}-5 ; \mathrm{Bl}=$ idioventricular arrhythmia in lead II; B2 = idioventricular arrhythmia in lead V-5; $\mathrm{Cl}=$ ventricular tachycardia in lead II; C2 = ventricular tachycardia in lead V-5; D = sinus tachycardia in lead II.

felt to be secondary to atelectasis and possibly central line placement without strict aseptic technique during resuscitation. She was placed on levofloxin for prophylactic antibiotic coverage. The patient was discharged to home on hospital day three neurologically intact, complaining only of mild musculoskeletal pain from the rib fractures.

\section{Discussion}

The diagnosis of AFE remains one of exclusion. Although the diagnosis of AFE was never confirmed histologically, secondary to the patient's survival, the presence of intra-uterine fetal demise with cardiopulmonary arrest and DIC implicate AFE as the most likely cause of this intraoperative event. AFE should be suspected in any pregnant patient, specifically those with ruptured membranes, who develop sudden onset dyspnea with hypoxia, acute hypotension and/or cardiac arrest followed by a profound coagulopathy. Both the United States and the United Kingdom have a national registry for suspected AFE, and entry criteria consist of the presence of the four following factors: 1 ) acute hypotension or cardiac arrest; 2) acute hypoxia; $3)$ coagulopathy or severe clinical hemorrhage in the absence of other explanations; 4 ) all of these occurring during labour, Cesarean delivery, or D \& $\mathrm{E}$ or within $30 \mathrm{~min}$ postpartum with no other explanation for the findings. ${ }^{1,4}$ The patient described in this case report had all of these factors. Lawson et al. ${ }^{5}$ reviewed all legal abortion related deaths from pulmonary embolism reported to the Centers for Disease Control (CDC) from 1972 through 1985. They found that embolism during legal induced abortion presents in a similar fashion as it does during term delivery, with sudden cardiovascular collapse, respiratory distress, cyanosis, and coma. ${ }^{5}$

The overall incidence of AFE has been reported to range from $l$ in 8,000 to $l$ in 80,000 deliveries. ${ }^{1,2}$ Although, other maternal catastrophes such as hemorrhage, pulmonary embolus and hypertensive disorders account for maternal deaths collectively, none are more devastating than AFE which has a maternal mortality rate that approaches 61 to $86 \%{ }^{1,2}$ AFE is responsible for about $10 \%$ of all maternal deaths in the United States and is the most common cause of peripartum death. ${ }^{1,2}$ Of the lethal dysrhythmias identified, $24 \%$ consisted of PEA, $22 \%$ of bradycardia, $17 \%$ of ventricular tachycardia/ventricular fibrillation, and $13 \%$ were asystole. ${ }^{1}$ The patient in this case report had many of the signs and symptoms associated with AFE including cough, dyspnea, hypotension and cardiac arrest. Interestingly, her potentially lethal cardiac rhythms included PEA, ventricular tachycardia, and asystole (Figure).

DIC develops in up to $83 \%$ of patients. ${ }^{1}$ DIC is a consumption coagulopathy, characterized by abnormal activation of the coagulation system resulting in formation of large amounts of thrombin, depletion of coagulation factors, activation of the fibrinolytic system and hemorrhage. ${ }^{6}$ In obstetrics, the most frequent causes of DIC are; AFE, retained dead fetus syndrome, pre-eclampsia, abruptio placentae, and sepsis. ${ }^{6}$ Laboratory findings consistent with DIC include; decreased platelet count, decreased fibrinogen and antithrombin III concentrations, variable increases in the INR, PT, and PTT, and increased concentrations of D-Dimer, fibrin monomer, and fibrin degradation products (Table I).

It was once thought that AFE resulted from powerful contractions that forced amniotic fluid into the maternal circulation through defects in the fetal membranes, placenta or elsewhere. ${ }^{2,7}$ Thus, women with tumultuous, hyper-stimulated, protracted labour were thought to be at greatest risk for developing AFE, and oxytocin and other uterine stimulants were also considered risk factors. ${ }^{2,7}$ However, analysis of the national registry by Clark et al. ${ }^{1}$ and the American college of Obstetricians and Gynecologists, ${ }^{8}$ have determined there is no causal relationship between oxytocin and AFE. Although the pathogenesis of AFE still remains poorly understood, more recent hypotheses have been proposed. Clark suggests that the hypertonic uterus 
TABLE I Preoperative, intraoperative, intensive care unit, postoperative and normal coagulation blood laboratory values

\begin{tabular}{|c|c|c|c|c|c|c|}
\hline & Preoperative & Intraoperative & $I C U$ & POD-1 & POD-2 & Normal range \\
\hline $\mathrm{Hb}\left(\mathrm{g} \cdot \mathrm{dL}^{-1}\right)$ & 10.6 & 9.2 & 10.2 & 9.8 & 10.1 & $11.6-14.6$ \\
\hline Platelets $\left(\times 10^{3}\right)$ & 391 & 214 & 211 & 183 & 256 & $156-369$ \\
\hline $\mathrm{PT}(\mathrm{sec})$ & 11.0 & 26.5 & 21.0 & 14.0 & 11.9 & $9.1-12.9$ \\
\hline $\operatorname{PTT}(\mathrm{sec})$ & 28.7 & 72.5 & 55.2 & 31.3 & 26.4 & $25.0-34.0$ \\
\hline INR & 1.0 & 2.39 & 1.90 & 1.27 & 1.08 & $0.9-1.1$ \\
\hline Fibrinogen $\left(\mathrm{mg} \cdot \mathrm{dL}^{-1}\right)$ & 467 & $<35$ & 39 & 136 & 281 & $200-445$ \\
\hline
\end{tabular}

ICU = intensive care unit; POD- $1=$ postoperative day 1 ; POD-2 = postoperative day $2 ; \mathrm{Hb}=$ hemoglobin; PT = prothrombin time; PTT $=$ partial thromboplastin time; INR $=$ international normalized ratio.

TABLE II Hemodynamic data

\begin{tabular}{|c|c|c|c|c|c|}
\hline & Preoperative & Pre-arrest & Arrest & Post-arrest & $I C U$ \\
\hline $\mathrm{SBP}(\mathrm{mmHg})$ & 110 & 105 & 88 & 158 & 136 \\
\hline DBP (mmHg) & 60 & 46 & 42 & 54 & 88 \\
\hline HR (beats. $\mathrm{min}^{-1}$ ) & 77 & 86 & 92 & 144 & 130 \\
\hline $\mathrm{SaO}_{2}(\%)$ & 99 & 99 & 84 & 98 & 100 \\
\hline
\end{tabular}

$\mathrm{ICU}=$ intensive care unit; $\mathrm{SBP}=$ systolic blood pressure; $\mathrm{DBP}=$ diastolic blood pressure; $\mathrm{HR}=$ heart rate; SaO $2=$ oxygen saturation.

observed with AFE is the physiologic response to a profound cardiovascular insult, including an outpouring of catecholamines, rather than the cause of the insult. ${ }^{1}$ Clark further states that these tetanic contractions elevate uterine pressures to pressures that would make maternal-fetal exchange via vascular communications unlikely. ${ }^{1}$

New theories suggest that AFE is actually a type 1 hypersensitivity reaction with mechanisms similar to anaphylaxis and septic shock, and that the variation in the nature and severity of the clinical syndrome appears to depend on the variation of the antigenic exposure and the individual response. ${ }^{l}$ Clark suggests that AFE should be renamed "Anaphylactoid Syndrome of Pregnancy." Forty-one (41\%) of the patients analyzed had a history of atopy or known drug allergies. ${ }^{1}$ Clark showed that $67 \%$ of those analyzed had been pregnant with a male fetus. ${ }^{1}$ Interestingly, the patient in this case report had a male fetus and she had a diagnosis of asthma with several antibiotic allergies.

Clark described a biphasic model for the pathogenesis of this proposed mechanism. Phase one is described as transient pulmonary vasospasm with release of vasoactive substances, which cause rightsided heart failure, hypotension, hypoxia and left ventricular injury. ${ }^{1}$ Progression to phase two results in left ventricular failure, pulmonary edema, and DIC. ${ }^{1}$

There is no one specific test that can confirm this syndrome. The postmortem diagnosis of AFE is often made at autopsy when fetal squames can be identified in the pulmonary vascular bed. ${ }^{1,2,9}$ The presence of fetal squamous cells in the pulmonary circulation was once thought to be pathognomonic for AFE. ${ }^{1,2,9}$ However, their presence alone does not confirm the diagnosis as fetal squames have been recovered from the pulmonary circulation in pregnant women who had undergone pulmonary artery catheterization for other reasons and showed no clinical evidence of AFE. ${ }^{9}$

In acute AFE, the initial presenting signs are often seen on the electrocardiogram and the pulse-oximeter. ${ }^{10}$ The electrocardiogram may show tachycardia with a right strain pattern and ST-T wave changes, and the pulse-oximeter may reveal a profound and sudden drop in $\mathrm{O}_{2}$ saturation. ${ }^{10}$ Shechtman suggests that transesophageal echocardgiograhy (TEE), used early in the insult, may help in establishing the diagnosis of the associated heart failure and may guide the therapy of this catastrophic condition. ${ }^{11}$ However, intraoperative TEE was not available at our institution, nor was it necessary in this case. Other investigators have looked at various markers that could rapidly detect and aid in the diagnosis of AFE. Determination of maternal plasma zinc copropophyrin (found in meconium), and the monoclonal antibody $\mathrm{TKH}-2$ have been reported as a means of rapid diagnosis of AFE. ${ }^{12,13}$ Future research regarding early diagnosis and possible prevention will certainly focus on cellular mediators such as histamine, prostaglandins, and leukotrienes whose biologic activity can explain many of the events that occur with AFE. 
The management of AFE remains largely supportive with emphasis on maintaining left ventricular function and output. ${ }^{10}$ Prompt recognition and institution of resuscitative measures may improve maternal and fetal outcome. CPR should be initiated immediately if indicated. If hypoxia refractory to $\mathrm{O}_{2}$ occurs or the patient becomes unconscious, the patient should be rapidly intubated and placed on $100 \% \mathrm{O}_{2}$ and mechanical ventilation. Maternal circulation should be supported with several large bore iv catheters and central access if possible. Initially, the goals are to rapidly increase the circulating volume and cardiac output with infusions of crystalloids, dopamine and other vasopressors as needed. Pulmonary artery and intraarterial catheters may be beneficial in guiding further therapy. If the patient is pregnant at the time of the AFE, plans should be made for immediate delivery after initial resuscitative efforts. Rapid delivery might not only improve fetal outcome, but may facilitate resuscitation particularly in term pregnancies. Because of the high maternal mortality rate, with more than half dying in the first hour, the surgeon should be prepared to perform a postmortem Cesarean section. Even though a $79 \%$ fetal survival rate has been reported, only $39 \%$ of these infants survive neurologically intact. ${ }^{l}$ Thus, clinicians should be prepared for immediate neonatal resuscitation. Coagulopathy also occurs in approximately $83 \%$ of cases, and is treated with component therapy including platelets, fresh frozen plasma, cryoprecipitate, and red blood cells. ${ }^{1}$ The administration of antifibrinolytics and blood products are best guided by the clinical situation and available hematological laboratory tests. Other more heroic measures including thrombectomy and cardiopulmonary bypass have been successful in the management of AFE. ${ }^{14}$ Oxytocin and injections of prostaglandin analogues as well as preparation for hypogastric artery ligation or hysterectomy may be necessary because of the high occurrence of uterine atony and postpartum hemorrhage in these patients. ${ }^{10,15}$ ICU care is crucial for the management of the sequelae of DIC and shock.

Since the pathophysiological mechanisms of AFE and anaphylaxis are similar, ${ }^{1}$ therapy directed at the management of AFE is analogous to the treatment of anaphylaxis. Primary treatment of anaphylaxis includes; stop all anesthetic agents, maintain an adequate airway and give $100 \% \mathrm{O}_{2}$, rapid $i v$ volume resuscitation, and epinephrine for cardiovascular support. ${ }^{16}$ In this case report, epinephrine was given via the endotracheal tube and is recommended by the American Heart Association when central venous access or peripheral iv access is poor or not available in providing ACLS. ${ }^{17}$ An advantage of endotracheal administration includes ease of administration and direct access into the pulmonary vasculature bypassing the right side of the heart. Even though pulmonary blood flow may be low during cardiac arrest, chest compression does provide blood flow and drug distribution to the heart. Secondary treatment includes the administration of; antihistamines $(\mathrm{Hl} / \mathrm{H} 2$ blockers), bronchodilators, corticosteroids and the maintenance of catecholamine infusions (epinephrine, norepinephrine, isoproterenol) to maintain hemodynamic stability. ${ }^{16}$ Indiscriminate prophylactic treatment to prevent anaphylaxis is not recommended and thus would not be indicated for the prevention of AFE. However primary and secondary treatment is indicated when necessary.

In summary, despite improvements in the recognition, monitoring and treatment of AFE both maternal and fetal mortality rate remain high. The case presented was most likely AFE given the clinical scenario and the events that followed. AFE can occur at anytime during gestation without warning. The syndrome remains unpredictable, unpreventable and often untreatable. The successful resuscitation presented in this report was the result of the rapid recognition, aggressive resuscitative efforts and the teamwork of health care personnel involved throughout this devastating and potentially catastrophic event.

\section{Acknowledgements}

The authors would like to thank Sheila Moffatt CRNA for her assistance in the resuscitation of the patient in this case report.

\section{References}

1 Clark SL, Hankins GD, Dudley DA, Dildy GA, Porter TF. Amniotic fluid embolism: analysis of the national registry. Am J Obstet Gynecol 1995; 172: 1158-69.

2 Morgan M. Amniotic fluid embolism. Anaesthesia 1979; 34: 20-32.

3 Cromey MG, Taylor PJ, Cumming DC. Probable amniotic fluid embolism after first-trimester pregnancy termination. A case report. J Reprod Med 1983; 28: 209-11.

4 de Swiet $M$. Maternal mortality: confidential enquiries into maternal deaths in the United Kingdom. Am J Obstet Gynecol 2000; 182: 760-6.

5 Lawson HW, Atrash HK, Franks AL. Fatal pulmonary embolism during legal induced abortion in the United States from 1972 to 1985. Am J Obstet Gynecol 1990; 162: 986-90.

6 Sharma SK, Lechner RB. Hematologic and coagulation disorders. In: Chestnut DH (Ed.). Obstetric

Anesthesia. Principles and Practice, 2nd ed. St. Louis: Mosby Inc.; 1999: 842-59. 
7 Steiner PE, Lushbaugh CC. Maternal pulmonary embolism by amniotic fluid. JAMA 1941; 117: 1340-5.

8 American College of Obstetricians and Gynecologists. Obstetric emergencies. In: Prolog: Obstetrics, 5 th ed. Washington, DC; 2003: 27.

9 Clark SL, Pavlova Z, Geenspoon J, Horenstein J, Phelan $J P$. Squamous cells in the maternal pulmonary circulation. Am J Obstet Gynecol 1986; 154: 104-6.

10 Martin RW. Amniotic fluid embolism. Clin Obstet Gynecol 1996; 39: 101-6.

11 Shechtman M, Ziser A, Markovits R, Rozenberg B. Amniotic fluid embolism: early findings of transesophageal echocardiography. Anesth Analg 1999; 89: 1456-8.

12 Kanayama N, Yamazaki T, Naruse H, Sumimoto K, Horiuchi $K$, Terao $T$. Determining zinc coproporphyrin in maternal plasma-a new method for diagnosing amniotic fluid embolism. Clin Chem 1992; 38: 526-9.
13 Kobayashi H, Obi H, Terao T. A simple, noninvasive, sensitive method for diagnosis of amniotic fluid embolism by monoclonal antibody TKH-2 that recognizes NeuAc $\alpha 2-6$ GalNAc. Am J Obstet Gynecol 1993; 168: 848-53.

14 Esposito RA, Grossi EA, Coppa G, et al. Successful treatment of postpartum shock caused by amniotic fluid embolism with cardiopulmonary bypass and pulmonary artery thromboembolectomy. Am J Obstet Gynecol $1990 ; 572-4$.

15 Hogberg U, Joelsson I. Amniotic fluid embolism in Sweden, 1951-1980. Gynecol Obstet Invest 1985; 20: 130-7.

16 Levy JH. The allergic response. In: Barash PG, Cullen BF, Stoelting RK (Eds.). Clinical Anesthesia, 3rd ed. Philadelphia: Lippincott-Raven Publishers; 1997: 1205-17.

17 Cummins RO. ACLS Provider Manual. Dallas: American Heart Association; 2001.

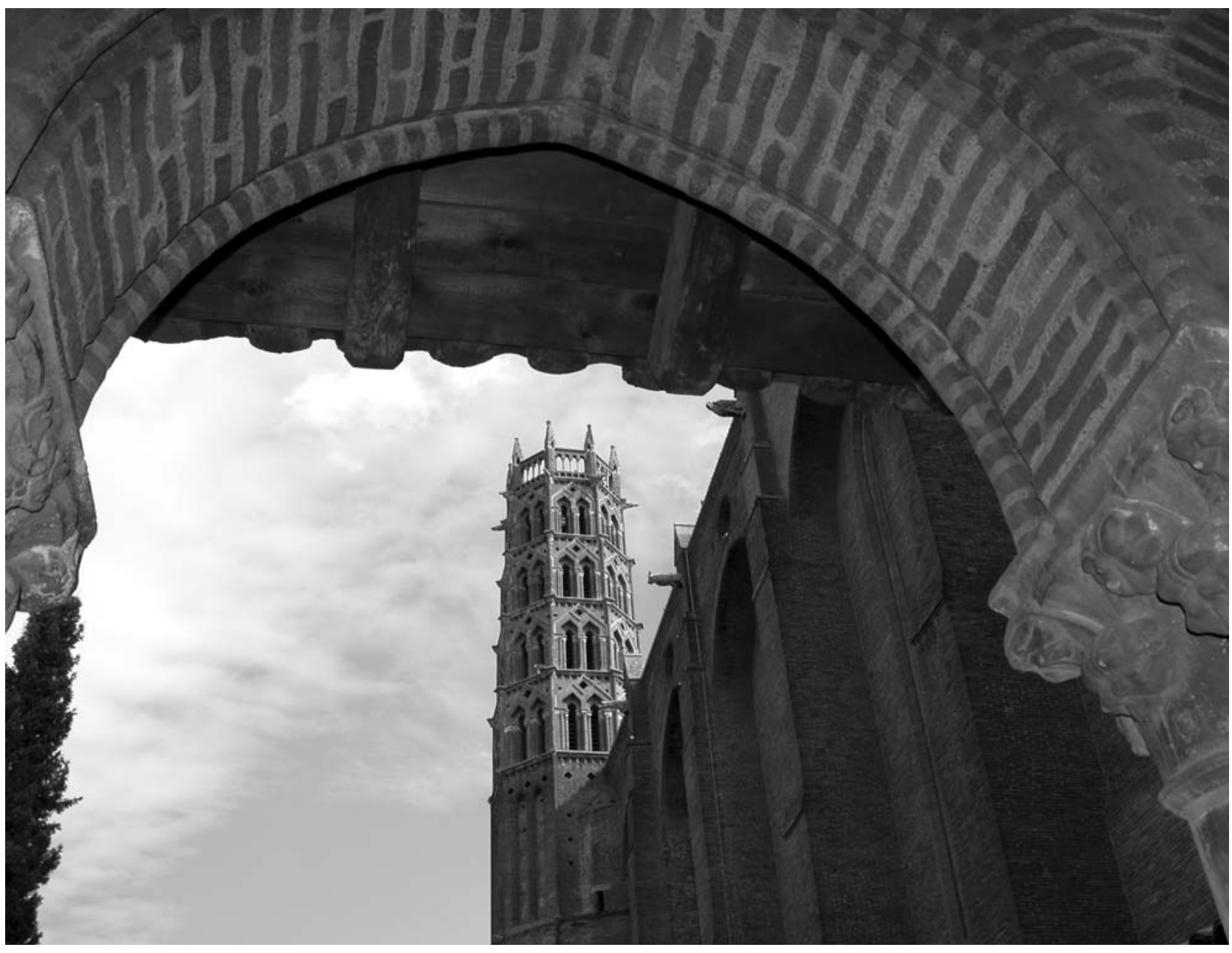

Cathédrale de Toulouse 